\title{
Attenuation of acute lung injury in a rat model by Semen Cassiae
}

Xiuqing Chen ${ }^{1^{* \dagger}}$, Xianming Zhang ${ }^{2+}$, Jie Zhang ${ }^{3}$, Yang Gao ${ }^{4}$, Zhaohui Yang ${ }^{5}$, Shanshan Li $^{1}$ and Haiwen Dai ${ }^{*^{*}}$

\begin{abstract}
Background: Acute lung injury (ALI) is an inflammatory disorder. Semen Cassiae has potent anti-inflammatory activities. The aim of our study was to investigate whether Semen Cassiae plays a protective effect on lipopolysaccharide (LPS)-induced ALI and, if so, to elucidate its potential mechanism.

Methods: Male Sprague-Dawley rat lungs were injured by intratracheal instillation of LPS. Rats were treated with Semen Cassiae or vehicle $3 \mathrm{~h}$ after LPS challenge. Samples were harvested $24 \mathrm{~h}$ post-LPS administration. We also investigated the effects of Semen Cassiae on LPS stimulation in RAW 264.7 cells.

Results: LPS administration markedly induced pulmonary edema and polymorphonuclear neutrophil influxes. These changes were significantly attenuated in Semen Cassiae treated group. Moreover, Semen Cassiae markedly reduced pulmonary interleukin (IL)-6, tumor necrosis factor (TNF)-a, and 8-hydroxy-2'-deoxyguanosine (8-OHdG) levels. The pulmonary soluble epoxide hydrolase (sEH) activity and the DNA binding activity of Nuclear factor (NF)-KB were significantly inhibited in Semen Cassiae treated group. Furthermore, Semen Cassiae treatment significantly increased epoxyeicosatrienoic acids (EETs), and heme oxygenase-1 (HO-1) activity. Our in vitro study demonstrates that Semen Cassiae treatment may inhibit LPS induced IKBa phosphorylation and NF-KB p65 nucleus translocation.

Conclusion: Semen Cassiae protects LPS-induced ALI in rats. Semen Cassiae can be developed as a novel treatment for ALI.
\end{abstract}

Keywords: Acute lung injury, Semen Cassiae, Inflammation

\section{Background}

Acute lung injury (ALI)/acute respiratory distress syndrome (ARDS) is severe disorder of lungs with high mortality $[1,2]$. The underlying mechanisms of ALI/ARDS are complex. It is suggested that systemic inflammatory response and oxidative stress play an important role in the development of ALI/ARDS [3]. Thus, it is logically to hypothesize that inhibiting inflammation and oxidative stress are effective strategy to reduce ALI/ARDS.

A recent study showed that soluble epoxide hydrolase $(\mathrm{sEH})$ plays a prevailing role in the pathogenesis of acute lung injury (ALI) [4]. sEH converted epoxyeicosatrienoic acids (EETs), an endothelium-derived factor with antiinflammatory effects, into dihydroxyeicosatrienic acids

\footnotetext{
*Correspondence: fortuneteller@163.com; c17177@163.com

${ }^{\dagger}$ Equal contributors

'Department of Emergency, Zhejiang Hospital, 12 Lingyin Road, Hangzhou

310013, China

Full list of author information is available at the end of the article
}

(DHET) [5]. Inhibition of sEH reduced cisplatin-induced renal apoptosis [6]. sEH inhibition has also shown protective effect on cardiomyocyte via EETs-dependent mechanisms [7]. Moreover, a sEH inhibitor reduced kidney ischemia-reperfusion injury by modulating inflammatory mediators [8]. These evidences suggest that $\mathrm{sEH}$ is a vital pharmacologic target for inflammation. Thus, drugs which have $\mathrm{sEH}$ inhibiting features may exert anti-inflammatory action.

Heme oxygenase (HO)-1, a rate-limiting enzyme in oxidative degradation of heme to biliverdin, plays an important effect in maintaining oxidative/antioxidant homeostasis [9]. Elevating HO-1 activity may reduce ALI $[10,11]$. It is now widely accepted that $\mathrm{HO}-1$ contributes to the antioxidative defenses in ALI $[10,11]$.

Semen Cassiae, the seeds of Cassia tora, contains multiple active ingredients such as anthraquinone compounds, cassiaside, and sitosterol. Semen Cassiae is a traditional Chinese medicine that has been 
demonstrated with anti-inflammatory and antioxidant features [12, 13]. Recent in vitro study demonstrated that Semen Cassiae has inhibitory effect on SEH [14]. Cui et al. reported that Semen Cassiae may reduce hepatic injury in rats [12]. However, it is still unknown that whether Semen Cassiae has any protective effect on ALI. The present study was conducted to test the hypothesis that Semen Cassiae attenuates lipopolysaccharide (LPS)-induced ALI through inhibition of inflammation and oxidative stress.

\section{Methods}

\section{Ethics statement}

All animal experiments were performed in accordance with the guidelines from the Administration of Animal Experiments for Medical Research Purposes issued by the Ministry of Health of China. The protocol used was reviewed and approved by the Animal Experiment Administration Committee of Zhejiang Hospital (Hangzhou, China).

\section{Animals}

Male Sprague-Dawley rats (160-180 g) were purchased from Zhejiang University (Hangzhou, China) and housed separately in a temperature controlled room on a $12 \mathrm{~h}$ light/12 h dark cycle. Animals received standard laboratory rodent chow ad libitum.

\section{Rats model of ALI}

All procedures were performed under anesthesia. Animals were randomly divided into various groups: control, LPS + vehicle, LPS + Semen Cassiae. Semen Cassiae was purchased from Shunchang herb store (Shanghai, China) and authenticated by Zhejiang Institute of Drug Control (Hangzhou, China). We obtained a crude decoction of Semen Cassiae by boiling $50 \mathrm{~g}$ Semen Cassiae in $500 \mathrm{~mL}$ distilled water for $2 \mathrm{~h}$. Then, the decoction was filtrated by a filter and concentrated to $50 \mathrm{~mL}$ by a low-pressure evaporator. A freeze-drying technique was used to process $5 \mathrm{~g}$ of Semen Cassiae powder through the resultant decoction. Before use, the Semen Cassiae powder was dissolved in phosphate buffered saline (PBS). In LPS + Semen Cassiae group, rats were fed once with $10 \mathrm{~g}$ of Semen Cassiae per kilogram of a rat $3 \mathrm{~h}$ after LPS administration. The dose of Semen Cassiae selected in our study was based on a previously published article [12]. Appropriate amount of the Semen Cassiae powder was dissolved in $1 \mathrm{~mL}$ PBS before use. Equivalent PBS was administrated for control group and LPS + vehicle group. Briefly, on the day of the experiment, animals were anesthetized by intraperitoneal injection of a cocktail of ketamine $(100 \mathrm{mg} / \mathrm{kg}) /$ xylazine ( $5 \mathrm{mg} / \mathrm{kg}$ ). Then, $50 \mu \mathrm{L}$ of LPS (Sigma, St. Louis, MO, USA) intratracheal instillation was performed to induce
ALI as described previously [15]. Before use, LPS was dissolved in sterilized PBS at $10 \mathrm{mg} / \mathrm{mL}$. We administrated equivalent PBS for control group. Rats in each group were sacrificed at $24 \mathrm{~h}$ after LPS or PBS challenge by cervical dislocation and exsanguinated by cutting the vena cava inferior. We homogenized lung tissue samples in PBS on ice to make the $10 \%$ pulmonary homogenate and stored at $-70{ }^{\circ} \mathrm{C}$ for further analysis.

\section{Pulmonary histological analysis}

Pulmonary specimen was fixed with $10 \%$ buffered formalin and stained with hematoxylin and eosin (H\&E). The degree of lung injury was scored by a histologist unaware of the sample groups. The degree of lung injury was scored according to: infiltration of neutrophils and thickness of the alveolar wall. Each assessment was graded as: 0, appears normal; 1, light; 2, moderate; 3, severe. We calculated a total score for each animal [16].

\section{The ratio of the lung wet to dry weight (W/D) analysis}

The lung was isolated and weighed before being dried in an oven at $80{ }^{\circ} \mathrm{C}$ for $48 \mathrm{~h}$. The dried lung was weighed again to calculate the pulmonary $\mathrm{W} / \mathrm{D}$ ratio.

\section{Measurement of sEH activity and EETs levels}

Pulmonary homogenate sEH activity and EETs levels were measured by an indirect method using a 14, 15-EET/DHET ELISA kit (Detroit R\&D, Michigan, USA) as described previously [4].

\section{Measurement of pulmonary inflammatory mediators}

Pulmonary homogenate interleukin (IL)-6 and tumor necrosis factor (TNF)- $\alpha$ were measured by use of a IL- 6 or TNF- $\alpha$ ELISA kit (R\&D Systems, Inc., USA) according to the manufacturers' manual.

\section{Determination of oxidative stress and HO-1 activity}

The level of 8-hydroxy-2'-deoxyguanosine (8-OHdG), an indicator of oxidative stress, was measured using ELISA kits (Biotechnology Co., Ltd. Shanghai, China) according to the manufacturers' manual.

An indirect method was used to detect the pulmonary homogenate HO-1 activity by measuring the rate of appearance of bilirubin as described previously [17].

\section{Nuclear factor (NF)-KB activity assay}

The DNA binding activity of NF- $\kappa B$ in the lung was determined by ELISA. An ELISA-based NF- $\mathrm{B}$ p50/p65 transcription factor assay kit (Chemicon, Temecula, CA) was used in the current study according to the manufacturer's instructions. 
Cell culture study and cell viability assay

We maintained RAW264.7 cells (the Cell Storehouse of the Chinese Academy of Science, Shanghai, China) in Dulbecco's modified Eagle's medium (DMEM) (Life Technologies, USA) at $37{ }^{\circ} \mathrm{C}$ under $5 \% \mathrm{CO}_{2}$. The DMEM contains $10 \%$ heat-inactivated fetal bovine serum (FBS), $100 \mathrm{U} / \mathrm{mL}$ penicillin, and $100 \mu \mathrm{g} / \mathrm{mL}$ streptomycin.

3-(4, 5-dimethylthiazol-2-yl)-2, 5-diphenyltetrazolium bromide (MTT) (Sigma Chemical Co. St. Louis, MO, USA) assay was used to determine cell cytotoxicity that caused by Semen Cassiae. In brief, RAW 264.7 cells $\left(5 \times 10^{4}\right.$ cells/well $)$ were treated with various concentrations of Semen Cassiae $(1 \mu \mathrm{mol} / \mathrm{L}, 10 \mu \mathrm{mol} / \mathrm{L}$ and $100 \mu \mathrm{mol} / \mathrm{L}$ ) and incubated in 48-well plates for $24 \mathrm{~h}$. Before use, the Semen Cassiae powder was dissolved in PBS. Equivalent PBS was administrated for control group. Then, $10 \mu \mathrm{L}$ of MTT solution $(5 \mathrm{mg} / \mathrm{mL})$ was added to each well of cells and further incubated for $4 \mathrm{~h}$. After the incubation, the supernatant was washed out. The insoluble formazan product was dissolved in $200 \mu \mathrm{L}$ of dimethyl sulfoxide (DMSO) for $15 \mathrm{~min}$, and the optical density (OD) at $570 \mathrm{~nm}$ was measured using a Microplate Reader. Cell viability in control medium was represented as $100 \%$.

\section{Western blotting}

The cells were pretreated with Semen Cassiae $(1 \mu \mathrm{mol} / \mathrm{L}$, and $10 \mu \mathrm{mol} / \mathrm{L})$ for $1 \mathrm{~h}$ and then the cells were stimulated with LPS $(1 \mu \mathrm{g} / \mathrm{mL})$ or PBS for $20 \mathrm{~h}$. Before use, the Semen Cassiae powder was dissolved in PBS. Equivalent PBS was administrated for control group and LPS + vehicle group. Nuclear and cytoplasmic protein from the supernatants was extracted for Western blot analysis using a Nuclear/Cytosol Extraction kit (BioVision, Inc., Mountain View, CA, USA). Rabbit NF- $\mathrm{B}$ p65 and $\mathrm{I} \kappa \mathrm{B} \alpha$ polyclonal antibodies (diluted 1:500; Santa Cruz Biotechnology; Santa Cruz, CA) were incubated with the blots overnight at $48{ }^{\circ} \mathrm{C}$. The blots were washed in TBST buffer. Then, secondary antibody, horseradish peroxidase-linked anti-rabbit IgG (Cell Signaling Technology, Danvers, $\mathrm{MA})$, were incubated with the blots for $1 \mathrm{~h}$ at room temperature. An anti-TATA antibody (diluted 1:1000; Abcam, Cambridge, MA, USA) and $\beta$-actin (diluted 1:200; Santa Cruz Biotechnology) were used as controls for nuclear and cytoplasmic proteins, respectively. We developed the blots by using an enhanced chemiluminescence detection kit (Amersham, Buckinghamshire, UK) and exposed on Hyperfilm ECL (Amersham, Buckinghamshire, UK). The NIH Image software (National Institutes of Health, Bethesda, MD, USA) was used to quantify protein band concentrations.

\section{Statistical analysis}

We presented all data as mean \pm SEM. All data were analyzed with the SPSS 17.0 program (IBM, Armonk, USA). $P<0.05$ was accepted as statistically significant. Differences between groups were carried out using oneway analysis of variance (ANOVA) followed by the Student-Newman-Keuls method. We performed histopathological scores by using Kruskal-Wallis one-way analysis of variance on ranks and the Student-NewmanKeuls method.

\section{Results}

\section{Effects of Semen Cassiae on ALI}

As shown in Fig. 1, LPS challenge significantly induced lung tissue histopathological changes characterized by lung edema, destruction of pulmonary architecture, alveolar hemorrhage, and neutrophil infiltration (Fig. 1a2). These changes were markedly improved by Semen Cassiae treatment (Fig. 1a3). Moreover, as indicated in Fig. 1b, the lung injury score was significantly dampened in Semen Cassiae treated group. The lung wet to dry weight ration, an indicator of pulmonary edema, was markedly attenuated by Semen Cassiae treatment (Fig. 1c).

\section{Effects of Semen Cassiae on pulmonary inflammatory mediators}

Pro-inflammatory cytokines play an important effect in the development of ALI/ARDS. In the present study, LPS challenge markedly increased pulmonary IL- 6 and TNF- $\alpha$ level (Fig. 2a, and b). Animals treated with Semen Cassiae had significantly lower IL-6 and TNF- $\alpha$ levels compared with vehicle treated group (Fig. 2a, and b).

\section{Effects of Semen Cassiae on sEH activity and EETs levels}

The activity of sEH was increased significantly in LPS + vehicle treated group compared with control (Fig. 2c). Semen Cassiae treatment markedly reduced the LPSinduced the elevation of sEH activity (Fig. 2c). EETs are known as an anti-inflammatory factor. In the present study, the EETs levels in pulmonary homogenate were significantly elevated in Semen Cassiae treated group compared with LPS + vehicle treated group (Fig. 2d).

\section{Effects of Semen Cassiae on 8-OHdG}

Pulmonary concentrations of $8-\mathrm{OHdG}$ were measured to investigate oxidative stress. 8-OHdG levels were markedly elevated in LPS + vehicle treated group (Fig. 3a). Semen Cassiae treatment significantly dampened 8-OHdG concentrations (Fig. 3a).

\section{Effects of Semen Cassiae on HO-1 activity}

HO-1 has antioxidant features. Our results showed an increase of HO-1 activity in pulmonary homogenate in 


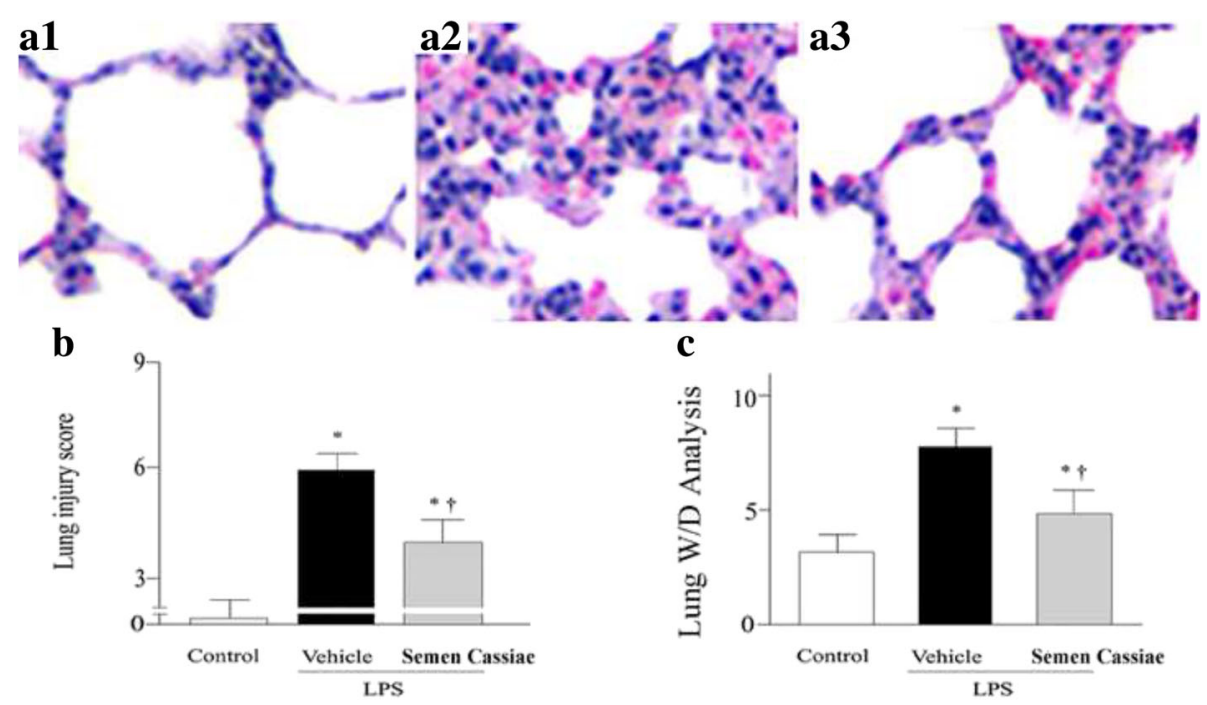

Fig. 1 Morphologic alterations of the lung were determined by photomicrography. a1) Photomicrograph of a pulmonary section from a rat of control group. a2) Photomicrograph of a pulmonary section from a rat of lipopolysaccharide (LPS) + Vehicle group. a3) Photomicrograph of a pulmonary section from a rat of LPS + Semen Cassiae group. Magnification: $\times 400$. Alterations of lung injury scores (b), or the ratio of the lung wet to dry weight (W/D) (c) in control or LPS challenged rats treated with vehicle or Semen Cassiae. Data are expressed as the mean \pm SEM and compared by one-way analysis of variance and the Student-Newman-Keuls method. We performed histopathological scores by using Kruskal-Wallis one-way analysis of variance on ranks and the Student-Newman-Keuls method. ${ }^{*} P<0.05$ when compared with control group; ${ }^{\dagger} P<0.05$ when compared with LPS + Vehicle group
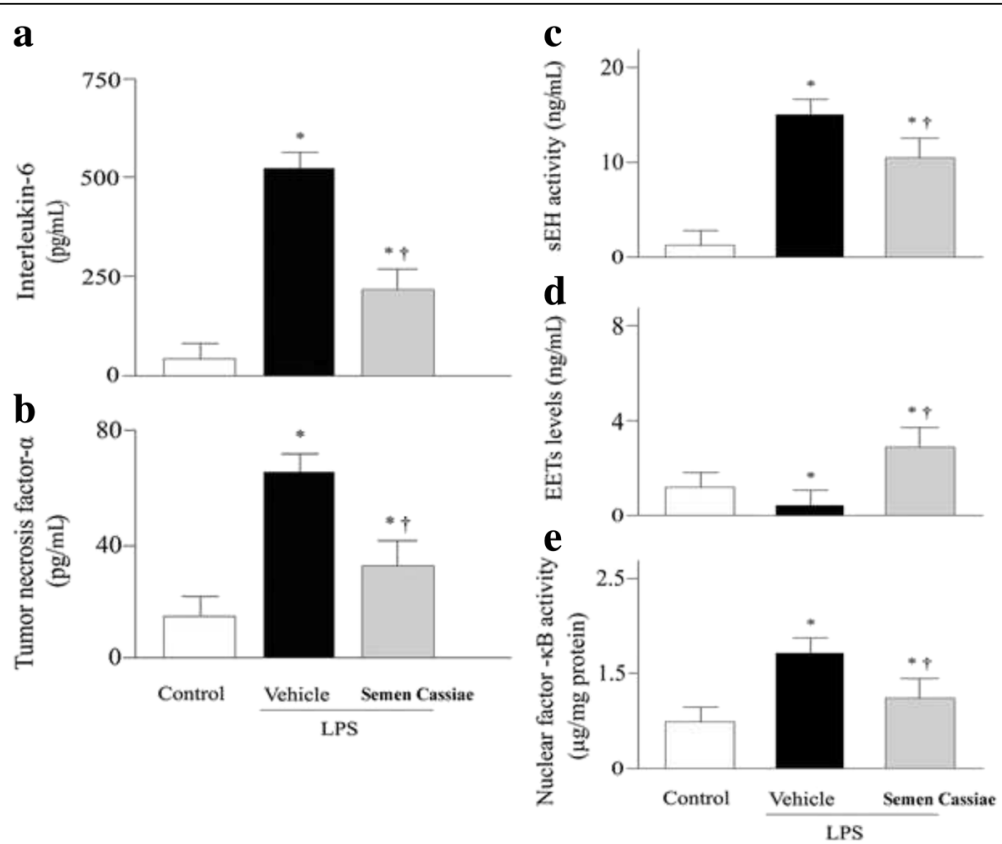

Fig. 2 Alterations of interleukin (IL)-6 (a), tumor necrosis factor (TNF)-a (b), soluble epoxide hydrolase (sEH) (c), epoxyeicosatrienoic acids (EETs) (d), and nuclear factor (NF)-KB DNA binding activity (e) in control or lipopolysaccharide (LPS) challenged rats treated with vehicle or Semen Cassiae. Data are expressed as the mean \pm SEM and compared by one-way analysis of variance and the Student-Newman-Keuls method. ${ }^{*} P<0.05$ when compared with control group; ${ }^{\dagger} P<0.05$ when compared with LPS + Vehicle group 


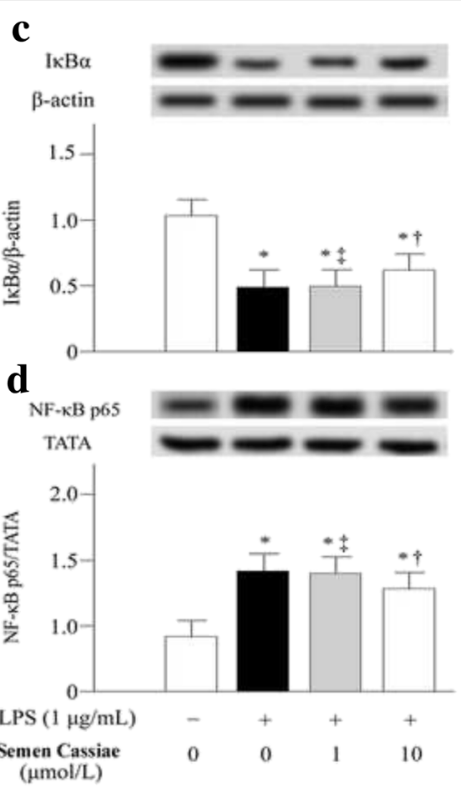

Fig. 3 Alterations of 8-hydroxy-2'-deoxyguanosine (8-OHdG) (a), and heme oxygenase (HO)-1 activity (b) in control or lipopolysaccharide (LPS) challenged rats treated with vehicle or Semen Cassiae. Alterations of IkBa levels in cytoplasm (c), and Nuclear factor (NF)-KB p65 levels in nuclear (d) in RAW264.7 cells in control or lipopolysaccharide (LPS) challenged group pretreated with vehicle or Semen Cassiae. Data are expressed as the mean \pm SEM and compared by one-way analysis of variance and the Student-Newman-Keuls method. ${ }^{*} P<0.05$ when compared with control group; $^{\dagger} P<0.05$ when compared with LPS + Vehicle group. ${ }^{\ddagger} P>0.05$ when compared with LPS + Vehicle group

LPS + vehicle treated group (Fig. 3b). This result suggests an endogenous anti-oxidant mechanism. However, this protective effect was limited, as marked increased 8-OHdG levels were observed in the LPS + vehicle treated group (Fig. 3a). Semen Cassiae treatment significantly increased HO-1 activity compared with LPS + vehicle treated group (Fig. 3b).

\section{Effects of Semen Cassiae on cell viability}

In order to test the cytotoxic effect of Semen Cassiae, the MTT assay was employed. Our results indicated that the viability of RAW 264.7 cells was not affected by Semen Cassiae treatment at concentrations up to $10 \mu \mathrm{mol} / \mathrm{L}$ (Additional file 1: Table S1).

\section{Effects of Semen Cassiae on NF-KB activation}

Expressions of numerous proinflammatory cytokines were regulated by $\mathrm{NF}-\mathrm{kB}$ which playing a vital role in the pathogenesis of ALI/ARDS [18-21]. We investigated the effect of Semen Cassiae on NF- $\mathrm{BB}$ activation in vivo and in vitro. Our Western blot analysis results indicated that the IкB $\alpha$ expression in the cytoplasm of RAW 264.7 cells was markedly decreased $20 \mathrm{~h}$ after LPS stimulation (Fig. 3c). Meanwhile, the NF-kB p65 in the nucleus of RAW 264.7 cells was significantly increased (Fig. 3d). Semen Cassiae inhibited the degradation of $\mathrm{I} \kappa \mathrm{B} \alpha$ and prevented the translocation of NF- $\mathrm{KB}$ into the nucleus in LPS stimulated RAW 264.7 cells (Fig. 3c, and d). Moreover, the NF- $\mathrm{KB}$ DNA binding activity in lungs was markedly inhibited in Semen Cassiae treated group compared with vehicle group (Fig. 2e).

\section{Discussion}

Semen Cassiae has anti-inflammatory and antioxidant features [12, 13]. However, the effect of Semen Cassiae on ALI, an inflammatory disorder, is still unclear. Our current study demonstrates the protective effect of Semen Cassiae on LPS-induced ALI characterized by reduced pulmonary edema and inflammation. The beneficial role of Semen Cassiae may be mediated via inhibiting $\mathrm{sEH}$ activity and NF- $\mathrm{kB}$ activation.

The current study indicates that Semen Cassiae treatment is associated with a decrease in sEH activity and elevating EETs levels. This result suggests a potential sEH inhibition activity of Semen Cassiae in vivo. Pharmacological inhibition of $\mathrm{sEH}$ has shown protective effect in different animal studies. Motoki et al. reported that $\mathrm{sEH}$ inhibition can reduce myocardial ischemiareperfusion injury [22]. Cisplatin-induced renal apoptosis was attenuated by a sEH inhibitor via EETs dependent mechanisms [6]. Moreover, LPS-induced ALI was dampened by a $\mathrm{SEH}$ inhibitor in a preclinical research [4]. In the present study, pulmonary inflammation and injury was significantly reduced in Semen Cassiae treated group. This result indicated a protective effect of Semen Cassiae on ALI. The benefit role of Semen Cassiae may be associated with $\mathrm{sEH}$ inhibition. It has been reported that the protective effect of $\mathrm{sEH}$ 
inhibition may be mediated through EETs [6]. Mitochondrial function of cardiomyocytes exposed to LPS was significantly improved by 14,15 -EET in an in vitro study [23]. And LPS-induced cardiomyocytes cytotoxicity can be reduced by 14, 15-EET through peroxisome proliferator-activated receptors $\gamma$ (PPAR $\gamma$ ) signaling pathway [23]. Evidence has shown that protective effects of EETs on cerebral $\mathrm{I} / \mathrm{R}$ injury is associated with phosphatidylinositol 3-kinase (PI3K)/Akt pathway and ATP-sensitive potassium (KATP) channels [24]. In our study, the EETs level was significantly increased in Semen Cassiae treated group. The increased EETs level may contribute to the protective effect of Semen Cassiae on ALI.

HO-1 is an inducible form of $\mathrm{HO}$ which plays an important role on reduction of reactive oxygen species (ROS) [25]. As oxidative stress is thought to play a vital effect in the pathogenesis of ALI [3], drugs have antioxidant features may play a protective effect on ALI. In the present study, HO-1 activity was significantly elevated in Semen Cassiae treated group. Moreover, the 8-OHdG levels, an indicator of oxidative stress, were markedly reduced in Semen Cassiae treated group. These results indicated an anti-oxidant action of Semen Cassiae. Previous studies have shown the anti-oxidant features of Semen Cassiae [13]. However, its potential mechanisms are still unclear. Studies have indicated that the expression of HO-1 protein and HO-1 activity could be induced by EETs [18]. In the current study, the EETs level was markedly increased in Semen Cassiae treated group. Thus, it seems reasonable to propose that Semen Cassiae increases HO-1 activity through inhibiting sEH and elevating EETs.

NF- $\mathrm{kB}$, a well-documented transcription factor, regulates expressions of numerous proinflammatory cytokines such as TNF- $\alpha$, and IL-6 [23]. It has been reported that NF- $\mathrm{kB}$ activity is increased in patients with ARDS [20, 21]. In animal studies, inhibition of NF- $k B$ activation can dampen ALI [26, 27]. Evidence has shown that following LPS stimulation, NF- $\mathrm{kB}$ was activated [19]. In the current study, we examined the effect of Semen Cassiae on NF-kB activation. Our Western blot analysis results shown that the degradation of $I_{\kappa} \mathrm{B} \alpha$ and the translocation of NF$\kappa \mathrm{B}$ p65 into the nucleus were markedly inhibited in Semen Cassiae pretreated RAW 264.7 cells. As Western blot analysis can not detect the activity of $N F-\kappa B$, we performed an ELISA-based method to detect the NF-kB DNA binding activity. In our animal studies, the NF- $\mathrm{kB}$ activity in pulmonary homogenate was significantly attenuated by Semen Cassiae treatment. Consistent with previous findings [26], inhibition of NF-kB was followed by decreased proinflammatory cytokines expression. NF- $\mathrm{kB}$ has been suggested as a key pharmacologic target for ALI [19]. The NF-kB inhibiting effect of Semen Cassiae may, in part, contribute to the reduced ALI in rats.

\section{Conclusions}

Semen Cassiae protects LPS-induced ALI in rats. Semen Cassiae can be developed as a novel treatment for ALI.

\section{Additional file}

Additional file 1: Table S1. Cell viability assay measured by 3-(4, 5-dimethylthiazol-2-yl)-2, 5-diphenyltetrazolium bromide (MTT) (DOC 25 kb)

\section{Acknowledgments}

The authors thank Dong Xiaotang and Zhang Yun for useful discussions and technical assistance.

\section{Funding}

None

Availability of data and materials

All the data supporting these findings is contained within this manuscript.

Authors' contributions

Participated in research design: CXQ, YZH, GY, ZXM, DHW. Conducted experiments: CXQ, ZJ, LSS. Performed data collection: CXQ, LSS. Performed data analysis: CXQ, YZH. Wrote the manuscript: CXQ. All authors read and approved the final manuscript.

\section{Competing interests}

The authors declare that they have no competing interests.

Consent for publication

Not applicable.

Ethics approval and consent to participate

The Institutional Animal Care and Use Committee of Zhejiang Hospital approved all rat protocols.

\section{Publisher's Note}

Springer Nature remains neutral with regard to jurisdictional claims in published maps and institutional affiliations.

\section{Author details}

${ }^{1}$ Department of Emergency, Zhejiang Hospital, 12 Lingyin Road, Hangzhou 310013, China. ${ }^{2}$ Department of Respiratory Medicine, First Affiliated Hospital of Guizhou Medical University, Guiyang 550004, China. ${ }^{3}$ Department of Respiratory Medicine, Henan Provincial People's Hospital, 7 Weiwu Road, Zhengzhou 450003, China. ${ }^{4}$ Department of Respiratory Medicine, Beijing Anzhen Hospital of the Capital Medical University, 2 Anzhen Road, Beijing 100029, China. ${ }^{5}$ Department of Respiratory Medicine, the First People's Hospital of Qingdao Economic \& Technological Development Area, 9 Huangpujiang Road, Qingdao 266000, China.

Received: 8 December 2016 Accepted: 20 April 2017

Published online: 28 April 2017

\section{References}

1. Suresh R, Kupfer $Y$, Tessler S. Acute respiratory distress syndrome. N Engl J Med. 2000;343(9):660-1.

2. Leaver SK, Evans TW. Acute respiratory distress syndrome. BMJ. 2007; 335(7616):389-94.

3. Tasaka S, Amaya F, Hashimoto S, Ishizaka A. Roles of oxidants and redox signaling in the pathogenesis of acute respiratory distress syndrome. Antioxid Redox Signal. 2008;10(4):739-53. 
4. Tao W, Li PS, Yang LQ, Ma YB. Effects of a soluble Epoxide Hydrolase inhibitor on Lipopolysaccharide-induced acute lung injury in mice. PLoS One. 2016;11(8):e0160359.

5. Wang Y, Wei X, Xiao X, Hui R, Card JW, Carey MA, Wang DW, Zeldin DC Arachidonic acid epoxygenase metabolites stimulate endothelial cell growth and angiogenesis via mitogen-activated protein kinase and phosphatidylinositol 3-kinase/Akt signaling pathways. J Pharmacol Exp Ther. 2005;314(2):522-32.

6. Liu Y, Lu X, Nguyen S, Olson JL, Webb HK, Kroetz DL. Epoxyeicosatrienoic acids prevent cisplatin-induced renal apoptosis through a p38 mitogenactivated protein kinase-regulated mitochondrial pathway. Mol Pharmacol. 2013;84(6):925-34.

7. Merkel MJ, Liu L, Cao Z, Packwood W, Young J, Alkayed NJ, Van Winkle DM. Inhibition of soluble epoxide hydrolase preserves cardiomyocytes: role of STAT3 signaling. Am J Physiol Heart Circ Physiol. 2010;298(2): H679-87.

8. Lee JP, Yang SH, Lee HY, Kim B, Cho JY, Paik JH, Oh YJ, Kim DK, Lim CS, Kim YS. Soluble epoxide hydrolase activity determines the severity of ischemiareperfusion injury in kidney. PLoS One. 2012;7(5):e37075.

9. Ryter SW, Alam J, Choi AM. Heme oxygenase-1/carbon monoxide: from basic science to therapeutic applications. Physiol Rev. 2006;86(2):583-650.

10. An L, Liu CT, Yu MJ, Chen ZH, Guo XG. Peng-Wang, Wang JF, fang XQ, Gao YH, Yu SY. Heme oxygenase-1 system, inflammation and ventilator-induced lung injury. Eur J Pharmacol. 2012;677(1-3):1-4.

11. Wu SY, Tang SE, Ko FC, Wu GC, Huang KL, Chu SJ. Valproic acid attenuates acute lung injury induced by ischemia-reperfusion in rats. Anesthesiology. 2015;122(6):1327-37.

12. Cui $X Y$. Protective effects of Semen Cassiae aqueous extract on acute hepatic injury in rats. J Med Sci Yanbian University. 2006;4:243-6.

13. Zhang XX, Zhang M, Wang Y, Miao F, Zhou L. Antioxidant activity of Cassid obtusifolia $\mathrm{L}$. seed in the process of germination. Acta Bot Boreal-Occident Sin. 2011;31:0393-7.

14. Lee GY, Kim JH, Choi SK, Kim YH. Constituents of the seeds of Cassia tora with inhibitory activity on soluble expoxide hydrolease. Bioorg Med Chem Lett. 2015;25(22):5097-101.

15. Lin WC, Lin CF, Chen CL, Chen CW, Lin YS. Inhibition of neutrophil apoptosis via sphingolipid signaling in acute lung injury. J Pharmacol Exp Ther. 2011;339(1):45-53.

16. Peng CK, Huang KL, Wu CP, Li MH, Hu YT, Hsu CW, Tsai SH, Chu SJ. Glutamine protects ischemia-reperfusion induced acute lung injury in isolated rat lungs. Pulm Pharmacol Ther. 2011;24(1):153-61.

17. Ryter SW, Kvam E, Tyrrell RM. Heme oxygenase activity. Current methods and applications. Methods Mol Biol. 2000;99:369-91.

18. Sacerdoti D, Colombrita C, Di Pascoli M, Schwartzman ML, Bolognesi M Falck JR, Gatta A, Abraham NG. 11,12-epoxyeicosatrienoic acid stimulates heme-oxygenase-1 in endothelial cells. Prostaglandins Other Lipid Mediat. 2007;82(1-4):155-61.

19. Adcock IM. Transcription factors as activators of gene transcription: AP-1 and NF-kappa B. Monaldi Arch Chest Dis. 1997:52(2):178-86.

20. Schwartz MD, Moore EE, Moore FA, Shenkar R, Moine P, Haenel JB, Abraham E. Nuclear factor-kappa B is activated in alveolar macrophages from patients with acute respiratory distress syndrome. Crit Care Med. 1996;24(8):1285-92.

21. Moine P, Mclntyre R, Schwartz MD, Kaneko D, Shenkar R, Le Tulzo Y, Moore EE, Abraham E. NF-kappaB regulatory mechanisms in alveolar macrophages from patients with acute respiratory distress syndrome. Shock. 2000;13(2):85-91.

22. Motoki A, Merkel MJ, Packwood WH, Cao Z, Liu L, lliff J, Alkayed NJ, Van Winkle DM. Soluble epoxide hydrolase inhibition and gene deletion are protective against myocardial ischemiareperfusion injury in vivo. Am J Physiol Heart Circ Physiol. 2008;295(5):H2128-34.

23. Samokhvalov V, Vriend J, Jamieson KL, Akhnokh MK, Manne R, Falck JR, Seubert JM. PPARY signaling is required for mediating EETs protective effects in neonatal cardiomyocytes exposed to LPS. Front Pharmacol. 2014;5:242.

24. Qu YY, Yuan MY, Liu Y, Xiao XJ, Zhu YL. The protective effect of epoxyeicosatrienoic acids on cerebral ischemia/reperfusion injury is associated with PI3K/Akt pathway and ATP-sensitive potassium channels. Neurochem Res. 2015:40(1):1-14.

25. Abraham NG, Drummond GS, Lutton JD, Kappas A. The biological significance and physiological role of heme oxygenase. Cell Physio Biochem. 1996;6:129-68.
26. Shu YS, Tao W, Miao QB, Zhu YB, Yang YF. Improvement of ventilationinduced lung injury in a rodent model by inhibition of inhibitory $\mathrm{kB}$ kinase. J Trauma Acute Care Surg. 2014;76(6):1417-24.

27. Li Y, Zeng Z, Cao Y, Liu Y, Ping F, Liang M, Xue Y, Xi C, Zhou M, Jiang W. Angiotensin-converting enzyme 2 prevents lipopolysaccharide-induced rat acute lung injury via suppressing the ERK1/2 and NF-KB signaling pathways. Sci Rep. 2016;6:27911.

\section{Submit your next manuscript to BioMed Central and we will help you at every step:}

- We accept pre-submission inquiries

- Our selector tool helps you to find the most relevant journal

- We provide round the clock customer support

- Convenient online submission

- Thorough peer review

- Inclusion in PubMed and all major indexing services

- Maximum visibility for your research

Submit your manuscript at www.biomedcentral.com/submit
) Biomed Central 